\title{
Representations of quantum orders
}

\author{
A. N. Panov \\ Russia, 443011, Samara, ul.Akad. Pavlova 1, Samara State University, \\ Mathematical Department \\ apanov@list.ru
}

1

\begin{abstract}
We study finite dimensional algebras that appear as fibers of quantum orders over a given point of variety of center. We present the formula for the number of irreducible representations and check it for it for the algebra of twisted polynomials, the quantum Weyl algebra and the algebra of regular functions on quantum group.
\end{abstract}

\section{$1 \quad$ Introduction and main statements}

Quantum algebras appear in the framework of mathematical physics. From the algebraic point of view a quantum algebra $R_{q}$ is an domain and a free $\mathbb{C}\left[q, q^{-1}\right]$-module. After specialization module $q-\varepsilon$ we obtain the algebra $R_{\varepsilon}=R_{q} \bmod (q-\varepsilon)$. As usual (see algebras A1-A4 below) $R_{\varepsilon}$ is a domain and, if $\varepsilon$ is a root of unity, then $R_{\varepsilon}$ is finite over its center $Z_{\varepsilon}$. We call $R_{\varepsilon}$ a quantum order (since it becomes an order in the skew field $R_{\varepsilon} \otimes \operatorname{Fract}\left(Z_{\varepsilon}\right)$ ). This algebra defines the affine variety $\mathfrak{X}=\operatorname{Maxspec} Z_{\varepsilon}$ that is singular in general.

The order $R_{\varepsilon}$ has one remarkable property: it admits the quantum adjoint action. For $a, u \in R_{q}$ we denote $a_{\varepsilon}, u_{\varepsilon}:=a, u \bmod (q-\varepsilon) \in R_{\varepsilon}$. If $u_{\varepsilon}$ lies in $Z_{\varepsilon}$, then the formula

$$
\mathcal{D}_{u}\left(a_{\varepsilon}\right)=\frac{u a-a u}{q-\varepsilon} \bmod (q-\varepsilon)
$$

defines the derivation $\mathcal{D}_{u}: R_{\varepsilon} \rightarrow R_{\varepsilon}$, that is called the quantum adjoint action of $u$ [1, 2, 4, 10, 11]. The center $Z_{\varepsilon}$ is a Poisson algebra with respect to the bracket $\left\{u_{\varepsilon}, v_{\varepsilon}\right\}:=\mathcal{D}_{u}\left(v_{\varepsilon}\right)=$ $-\mathcal{D}_{v}\left(u_{\varepsilon}\right)$. The variety $\mathfrak{X}=\operatorname{Maxspec} Z_{\varepsilon}$ is a Poisson variety. It splits into symplectic leaves [7].

For a point $\chi \in \mathfrak{X}=\operatorname{Maxspec} Z_{\varepsilon}$, we consider the finite dimensional algebra $R_{\chi}=R_{\varepsilon} / R_{\varepsilon} m_{\chi}$. Call it the fiber of $R_{\varepsilon}$. An irreducible representation of $R_{\varepsilon}$ with central character $\chi$ passes through $R_{\varepsilon} \rightarrow R_{\chi}$. Therefore, these representations are in one to one correspondence with irreducible representations of $R_{\chi}$

The goal of this paper is to characterize the fibers in terms of a point $\chi$ of Poisson variety $\mathfrak{X}$. Main Theorem will be proved in the case when $R_{q}$ is one of the following algebras: A1) Algebra of twisted polynomials, A2) Quantum Weyl Algebra, A3) $U_{q}(\mathfrak{b})$ (this algebra is isomorphic to $\mathbb{C}_{q}[B]$ for the Borel subroup $\left.B\right)$, A4) algebra $\mathbb{C}_{q}[G]$ of regular functions on the quantum semisimple Lie group $G$. For definitions see e.g. 6]

\footnotetext{
${ }^{1}$ The paper is supported by the RFFI-grants 02-01-00017 and 03-01-00167.
} 
We introduce the notion of stabilizer for any point of commutative associative Poisson $\mathbb{C}$-algebra $\mathcal{F}$. Recall that an algebra $\mathcal{F}$ is a Poisson algebra if it admits a Poisson bracket that is a linear, skew-symmetric map $\{\cdot, \cdot\}: \mathcal{F} \otimes \mathcal{F} \rightarrow \mathcal{F}$, subjecting to the Jacobi and the Leibniz identity (i.e., $\{a, b c\}=\{a, b\} c+b\{a, c\}$ for all $a, b, c \in \mathcal{F}$ ). A Poisson algebra is a Lie algebra with respect to the Poisson bracket. We call $P$ an ideal(resp. Poisson ideal) of Poisson algebra $\mathcal{F}$ if $P$ is an ideal of commutative associative algebra $\mathcal{F}$ (resp. $P$ is an ideal of $\mathcal{F}$ and $\{P, \mathcal{F}\} \subset P)$. We identify a point $\chi \in \mathfrak{X}=\operatorname{Maxspec} \mathcal{F}$ with the character $\chi: \mathcal{F} \rightarrow \mathbb{C}$. We use the notation $m_{\chi}$ for the corresponding maximal ideal in $\mathcal{F}$. The subalgebra

$$
G_{\chi}:=\left\{a \in \mathcal{F}:\{a, \mathcal{F}\} \in m_{\chi}\right\}
$$

is a Poisson subalgebra of $\mathcal{F}$. The ideal $m_{\chi}^{2}$ is contained in $G_{\chi}$ and is a Poisson ideal in $G_{\chi}$. Definition 1.1. The finite dimensional Lie $\mathbb{C}$-algebra

$$
\mathfrak{g}_{\chi}:=G_{\chi} / m_{\chi}^{2}
$$

is called the stabilizer of the point $\chi \in \mathfrak{X}$. If $\mathcal{F}$ is generated (as commutative associative $\mathbb{C}$ algebra) by $a_{1}, \ldots, a_{N}$, then $\mathfrak{g}$ is the linear span of $\overline{a_{i}}:=a_{i}-\chi\left(a_{i}\right) \bmod m_{\chi}^{2}$. The definition of stabilizer in the case of smooth manifolds is given in [8].

If $\mathfrak{g}$ is a finite dimensional Lie algebra over $\mathbb{C}$ and $\mathfrak{n}$ is the maximal nilpotent ideal (i.e., nilradical) of $\mathfrak{g}$, then the Lie algebra $\mathfrak{g} / \mathfrak{n}$ is a reductive algebra. We denote by rankg the dimension of maximal commutative subalgebra in $\mathfrak{g} / \mathfrak{n}$. If $\mathfrak{g}$ is an algebraic solvable Lie algebra (i.e., the Lie algebra of some algebraic solvable $\mathbb{C}$-group $\mathfrak{G}$ ), then $\mathfrak{g}=\mathfrak{t} \oplus \mathfrak{n}$ where $\mathfrak{t}$ is a toroidal Lie subalgebra of $\mathfrak{g}$. In this case $\operatorname{dim}_{\mathbb{C}} \mathfrak{t}=$ rankg.

Recall the definition of Ore extension. Given an algebra $A$; an automorphism $\tau: A \rightarrow A$ and a $\tau$-derivation $\delta: A \rightarrow A$ (i.e. $\delta(a b)=\delta(a) b+\tau(a) \delta(b)$ for all $a, b \in A$ ). An algebra $R$ is an Ore extension of $A$ if $R$ is generated by $A$ and an indeterminate $x$ with the defining relations $x a=\tau(a) x+\delta(a)$ for all $a \in A$ [6, 12].

Let we have a skew-symmetric integer matrix $\mathbb{S}=\left(s_{i j}\right)_{i j=1}^{N}$ and an indeterminate $q$. Put $q_{i j}=q^{s_{i j}}$ and form the matrix $\mathbb{Q}=\left(q_{i j}\right)_{i j}^{n}$. By definition, an algebra $R_{q}$ is a quantum solvable algebra over $C:=\mathbb{C}\left[q, q^{-1}\right]$ if it is generated by the elements $x_{1}, x_{2}, \ldots, x_{n}, x_{n+1}^{ \pm 1}, \ldots, x_{N}^{ \pm 1}$ and $C$ and such that any its subalgebra

$$
R_{i}:=<x_{i}, \ldots, x_{n}, x_{n+1}^{ \pm 1}, \ldots, x_{N}^{ \pm 1}, C>, \quad 1 \leq i \leq n
$$

is an Ore extension $\left.R_{i}=R_{i+1}\left[x_{i} ; \tau_{i}, \delta_{i}\right]\right)$ with $\tau\left(x_{j}\right)=q_{i j} x_{j}, \quad i+1 \leq j \leq N$ and $x_{i} x_{j}=q_{i j} x_{j} x_{i}$, $1 \leq i \leq N, n+1 \leq j \leq N$ [9, 11]. All algebras A1-A4 are quantum solvable algebras (see [9, 11]). More precisely, the algebra $\mathbb{C}_{q}[G]$ becomes quantum solvable after some localization (see section 3). In what follows we suppose that all quantum solvable algebras obey the following conditions.

1) " $q$-skew condition": $\tau_{i} \delta_{i}=q^{s_{i}} \delta_{i} \tau_{i}$ with some $s_{i} \in \mathbb{Z}$; assume that $s_{i} \neq 0$ for $\delta_{i} \neq 0$ and $s_{i}=0$ for $\delta_{i}=0$. Call the system of non-zero integers $\left\{s_{i}\right\}$ the system of exponents of $R_{q}$;

2) All $\delta_{i}$ is locally nilpotent.

Notice that the algebras A1-A4 obey these two conditions.

Let $\varepsilon$ be a primitive $l$ th root of unity. For the algebras A1-A2 we call $l$ (and $\varepsilon$ ) admissible if if $l$ is relatively prime with all principal minors of $\mathbb{S}$ and with the system of exponents $s_{1}, \ldots, s_{N}$. For the algebras A3-A4 $l$ (and $\varepsilon$ ) is admissible if $l$ is odd and $l \geq 3$ in the case $G$ has $\mathrm{G}_{2}$ components.

If $R_{q}$ is one of the algebras A1-A4 and $l$ is an admissible root of unity, then the elements $a_{i}:=x_{i, \varepsilon}^{l} 1 \leq i \leq N$ (here $\left.x_{i, \varepsilon}=x_{i} \bmod (q-\varepsilon)\right)$ lie in the center $Z_{\varepsilon}$ of $R_{\varepsilon}$ [11, Lemma 2.19]. 
The central subalgebra $Z_{0}$, generated by $a_{i}, 1 \leq i \leq n$ and $a_{i}^{-1}, n+1 \leq i \leq N$, is isomorphic to $\mathbb{C}\left[a_{1}, \ldots, a_{n}, a_{n+1}^{ \pm 1}, \ldots a_{N}^{ \pm 1}\right]$. The subalgebra $Z_{0}$ is called the $l$-center of $R_{\varepsilon}$. For all algebras A1-A4 the subalgebra $Z_{0}$ is a Poisson subalgebra of $Z_{\varepsilon}$. The embedding $Z_{0} \subset Z_{\varepsilon}$ defines the projection $\phi: \mathfrak{X} \rightarrow \mathfrak{X}_{0}$ where $\mathfrak{X}_{0}=$ Maxspec $Z_{0}$.

The goal of this paper is to prove the following statement for the algebras A1-A4.

Main Theorem. Let $R$ be one of the algebras A1-A4. Suppose that $l$ is an admissible. Let $\phi: \chi \mapsto \chi_{0}$ and $\mathfrak{g}_{\chi}\left(\right.$ resp. $\left.\mathfrak{g}_{\chi_{0}}\right)$ are stabilizer of $\chi\left(\right.$ resp. $\left.\chi_{0}\right)$. Then

1) $\mathfrak{g}_{\chi}$ and $\mathfrak{g}_{\chi_{0}}$ are algebraic solvable Lie algebras with decompositions $\mathfrak{g}_{\chi}=\mathfrak{t} \oplus \mathfrak{n}$ and $\mathfrak{g}_{\chi 0}=\mathfrak{t}_{0} \oplus \mathfrak{n}_{0}$.

2) the subalgebra $G_{\chi_{0}}$ (of the algebra $Z_{0}$ ) is contained in $G_{\chi}$; the embedding $G_{\chi_{0}} \subset G_{\chi}$ is extended to the homomorphism $\psi_{p}: \mathfrak{g}_{\chi_{0}} \rightarrow \mathfrak{g}_{\chi}$ such that its restriction on $\mathfrak{t}_{0}$ is an isomorphism $\left.\psi_{p}\right|_{\mathfrak{t}_{0}}: \mathfrak{t}_{\chi_{0}} \rightarrow \mathfrak{t}_{\chi} ; \operatorname{rank} \mathfrak{g}_{\chi}=\operatorname{rankg}_{\chi_{0}}$

3) the number $\left|\operatorname{Irr} R_{\chi}\right|$ of irreducible representations of $R_{\varepsilon}$ with central character $\chi$ is equal to $l^{\text {rankg }}$.

In the section 2 we prove Main Theorem in the partial case (Proposition 2.2) and show that Main Theorem is true for the quantum solvable algebras with "admissible stratification" (Proposition 2.5). In the next section 3 we prove the existence of admissible stratifications for the algebras A1-A4. This will conclude the proof of Main Theorem for the algebras A1-A4 (see Propositions 3.1-3.4).

\section{Standard ideals}

Let $\mathbb{S}=\left(s_{i j}\right)_{i j=1}^{N}, C:=\mathbb{C}\left[q, q^{-1}\right]$ be as above. We denote by $A_{\mathbb{Q}}$ the algebra of twisted polynomials (see section 3 ).

Consider a quantum solvable algebra $\mathfrak{R}^{\prime}$ over $C$, generated by

$$
x_{1}, \ldots, x_{m}, x_{m+1}, \ldots, x_{N}
$$

Suppose that the elements $x_{1}, \ldots, x_{m}$ are $q$-commute, i.e. $x_{i} x_{j}=q_{i j} x_{j} x_{i}, 1 \leq i, j \leq m$. The multiplicatively closed subset $\mathfrak{S}$, generated by $x_{1}, \ldots, x_{m}$, is a denominator subset [17, Lemma 2.1]. Denote $\mathfrak{R}:=\mathfrak{R}^{\prime} \mathfrak{S}^{-1}$. There exist the elements $\tilde{x}_{m+1}, \ldots, \tilde{x}_{N}$ in the localization of $\Re S_{*}^{-1}$ (here $S_{*}$ is finitely generated, by some $\left\{q^{i}-1\right\}$, denominator subset in $C$ ) such that $x_{i} \tilde{x}_{j}=q_{i j} \tilde{x}_{j} x_{i}, 1 \leq i \leq m, m+1 \leq j \leq N$. The subalgebra, generated by $\tilde{x}_{m+1}, \ldots, \tilde{x}_{N}$ coincides with $\mathfrak{R}_{m+1}=<x_{m+1}, \ldots, x_{N}>$ [17, Prop.2.1-2.3]. Suppose that the ideal $\mathfrak{I}^{\prime}$ ( of $\mathfrak{R} S_{*}^{-1}$ ), generated by $\tilde{x}_{j}, m+1 \leq j \leq N$, has zero intersection with $C$. Denote $\mathfrak{I}:=\mathfrak{I}^{\prime} \cap \mathfrak{R}$. Call $(\mathfrak{R}, \mathfrak{I})$ a standard pair and $\mathfrak{I}$ a standard ideal in $\mathfrak{R}$. Notice that the subalgebra $\mathfrak{B}$, generated over $C$ by $x_{i}, 1 \leq i \leq m$, is an algebra of twisted Laurent polynomials and $\mathfrak{B}=\mathfrak{R} / \mathfrak{I}$.

Let $\varepsilon$ be a primitive $l$ th root of unity and $l$ is relatively prime with all principal minors of $\mathbb{S}$. We denote by $\mathfrak{Z}_{\varepsilon}$ the center of $\mathfrak{R}_{\varepsilon}:=\mathfrak{R} \bmod (q-\varepsilon)$ and $\mathfrak{X}:=\operatorname{Maxspec} \mathfrak{Z}_{\varepsilon}$. The elements $a_{i}:=x_{i, \varepsilon}^{l}, 1 \leq i \leq m$ lie in $\mathfrak{Z}_{\varepsilon}$. Let $\mathfrak{Z}_{0}$ be some subalgebra of $\mathfrak{Z}_{\varepsilon}$ such that $\mathfrak{Z}_{0} \cap \mathfrak{B}_{\varepsilon}$ is generated by $a_{i}:=x_{i, \varepsilon}^{l}, 1 \leq i \leq m$ and $\mathfrak{R}_{\varepsilon}$ is finite over $\mathfrak{Z}_{0}$. The center $\mathfrak{Z}_{\varepsilon}$ is finite over $\mathfrak{Z}_{0}$. Denote $\mathfrak{X}_{0}:=\operatorname{Maxspec} \mathfrak{Z}_{0}, \mathfrak{I}_{\varepsilon}=(\mathfrak{I}+\mathfrak{R}(q-\varepsilon)) \bmod (q-\varepsilon), \phi: \mathfrak{X} \rightarrow \mathfrak{X}_{0}, \mathfrak{i}:=\mathfrak{I}_{\varepsilon} \cap \mathfrak{Z}_{\varepsilon}, \mathfrak{i}_{0}:=\mathfrak{I}_{\varepsilon} \cap \mathfrak{Z}_{0}$.

The skew field Fract $(\mathfrak{R})$ is isomorphic to the skew field Fract $\left(A_{\mathbb{Q}}\right)$ of the algebra $A_{\mathbb{Q}}(=\operatorname{gr}(\Re))$ of twisted polynomials (see section 3) [9, 11, 17]. We are going to prove Main Theorem for the case $\chi$ (resp. $\left.\chi_{0}\right)$ is a point of $\mathfrak{X}\left(\right.$ resp. $\left.\mathfrak{X}_{0}\right)$ annihilated by $\mathfrak{i}\left(\right.$ resp. $\left.\mathfrak{i}_{0}\right)$.

The algebra $\mathfrak{B}$ has a new system of generators $h_{i}, g_{i}, 1 \leq i \leq k$ and $z_{j}, 1 \leq j \leq p$, $2 k+p=m$ (that consists of monomials of $x_{i}^{ \pm 1}, 1 \leq i \leq m$ ) such that $h_{i} g_{i}=q^{d_{i}^{\prime}} g_{i} h_{i}$ and $\left\{z_{j}\right\}$ generate the center of $\mathfrak{B}$. By assumption, $l$ is relatively prime with $d_{i}^{\prime}$. The intersection of the center $\mathfrak{Z}$ of $\mathfrak{R}$ with $\mathfrak{B}$ is generated by some monomials $\left\{z^{a}:=z_{1}^{\alpha_{1}} \cdots z_{p}^{\alpha_{p}}, \alpha_{j} \in \mathbb{Z}\right\}$. Choosing the 
compatible basis, we may consider that the intersection $\mathfrak{Z} \cap \mathfrak{B}$ is generated by $z_{t+1}^{n_{t+1}}, \ldots, z_{p}^{n_{p}}$ for some $n_{t+1}, \ldots, n_{p} \in \mathbb{N}$. Since the field $\operatorname{Center}\left(\operatorname{Fract}\left(\left(\mathrm{A}_{\mathbb{Q}}\right)\right)\right.$ is algebraically closed in Fract $\left(A_{\mathbb{Q}}\right)$, then if an element $z^{d}$ also lies in the center of Fract $\left(A_{\mathbb{Q}}\right)$ (that is isomorphic to Fract $\left.(\Re)\right)$, then $z$ lies in the center. This verifies that $n_{t+1}=\cdots=n_{p}=1$.

Lemma 2.1.

1) The intersection $\mathfrak{Z}_{\varepsilon} \cap \mathfrak{B}_{\varepsilon}$ is generated by

$$
h_{i, \varepsilon}^{l}, \quad g_{i, \varepsilon}^{l}, \text { where } 1 \leq i \leq k \text { and } z_{1, \varepsilon}^{l}, \ldots, z_{t, \varepsilon}^{l}, z_{t+1, \varepsilon}, \ldots, z_{m, \varepsilon}
$$

2) The intersection $\mathfrak{Z}_{0} \cap \mathfrak{B}_{\varepsilon}$ is generated by

$$
h_{i, \varepsilon}^{l}, \quad g_{i, \varepsilon}^{l} \text { where } 1 \leq i \leq k \text { and } z_{1, \varepsilon}^{l}, \ldots, z_{t, \varepsilon}^{l}, z_{t+1, \varepsilon}^{l}, \ldots, z_{m, \varepsilon}^{l} .
$$

Proof. The statement 2) is trivial. To prove 1) it suffices to show that the monomial $z_{1, \varepsilon}^{\alpha_{1}} \ldots z_{t, \varepsilon}^{\alpha_{t}}$ lies in $\mathfrak{Z}_{\varepsilon}$ whenever $l$ divides all $\alpha_{i}$.

There exists the system of generators $\tilde{x}_{k_{1}}, \ldots, \tilde{x}_{k_{t}}$ in $A_{\mathbb{Q}}$ such that

$$
z_{j} \tilde{x}_{k_{j}}=q^{\nu_{i, k_{j}}} \tilde{x}_{k_{j}} z_{j} \quad \text { and } \mathrm{F}:=\operatorname{det}\left(\nu_{\mathrm{i}, \mathrm{k}_{\mathrm{j}}}\right)_{\mathrm{i}, \mathrm{j}=1}^{\mathrm{t}} \neq 0 \text {. }
$$

Connect $\tilde{x}_{k_{1}}, \ldots, \tilde{x}_{k_{t}}$ to the system $\left\{x_{i}, 1 \leq i \leq m\right\}$. Denote by $\mathbb{S}^{\prime \prime}$ the corresponding $(m+t) \times$ $(m+t)$-submatrix of $\mathbb{S}$. The rank of $\mathbb{S}^{\prime \prime}$ is equal to $2 k+2 t$ and the greatest common divisor $D^{\prime \prime}$ of all its $(2 k+2 t) \times(2 k+2 t)$-minors is equal to $\left(d_{1}^{\prime}\right)^{2} \ldots\left(d_{k}^{\prime}\right)^{2} F^{2}$. Since $l$ is admissible, $l$ relatively prime with $D^{\prime \prime}$. Therefore, $G C D(l, F)=1$. There exist $v_{i} \in \operatorname{Fract}(\mathfrak{R})$ such that

$$
z_{i} v_{j}=q^{p_{i} \delta_{i j}} v_{j} z_{i}, \text { and } \operatorname{GCD}\left(l, p_{i}\right)=1
$$

for all $1 \leq i, j \leq t$. This implies that, if $z_{1, \varepsilon}^{\alpha_{1}} \ldots z_{t, \varepsilon}^{\alpha_{t}}$ lies in $\mathfrak{Z}_{\varepsilon}$, then $l$ divides all $\alpha_{i}$.

Proposition 2.2. Let $\mathfrak{R}, \mathfrak{B}, \mathfrak{I}, \varepsilon$ be as above. Suppose that $\mathfrak{Z}_{0}$ is a Poisson subalgebra in $\mathfrak{Z}_{\varepsilon}$. Let $\chi \in \mathfrak{X}$ and $\chi_{0}=\phi(\chi) \in \mathfrak{X}_{0}$. Suppose that $\chi\left(\right.$ resp. $\left.\chi_{0}\right)$ is annihilated by the ideal $\mathfrak{i}$ (resp. $\left.\dot{i}_{0}\right)$ and $\chi\left(a_{i}\right) \neq 0,1 \leq i \leq m$. Then

1) the number of irreducible representations of $\mathfrak{R}_{\varepsilon}$ with central character $\chi$ is equal to $l^{t}$;

2 ) the ideal $\mathfrak{i}$ (resp. $\mathfrak{i}_{0}$ ) is a Poisson ideal in $G_{\chi}$ (resp. $G_{\chi_{0}}$ ). Denote by $\mathfrak{n}^{\prime}$ (resp. $\mathfrak{n}_{0}^{\prime}$ ) the image of $\mathfrak{i}$ (resp. $\left.\mathfrak{i}_{0}\right)$ in $\mathfrak{g}_{\chi}$ (resp. $\left.\mathfrak{g}_{\chi 0}\right)$;

3) the ideal $\mathfrak{n}^{\prime}$ (resp. $\mathfrak{n}_{0}^{\prime}$ ) is a nilpotent ideal in $\mathfrak{g}_{\chi}$ (resp. $\mathfrak{g}_{\chi 0}$ ). Then Main Theorem is true for $\mathfrak{R}$ and $\chi$. In particular, $\mathfrak{g}_{\chi}$ (resp. $\mathfrak{g}_{\chi_{0}}$ ) is an algebraic solvable Lie algebra.

Proof. First, notice that $\mathfrak{I}$ lies in the radical of $\mathfrak{R}_{\varepsilon} \mathfrak{i}_{0}$ (apply [11, Lemma 5.1]). Kernel of any irreducible representation $\pi$ with $l$-central character $\chi_{0}$ contains $\mathfrak{I}$. Any irreducible representation with $l$-central character $\chi_{0} \in \mathfrak{X}_{0}$ is uniquely determined by its kernel, generated by

$$
\begin{gathered}
\mathfrak{i}, \quad h_{i, \varepsilon}^{l}-\chi\left(h_{i, \varepsilon}^{l}\right), g_{i, \varepsilon}^{l}-\chi\left(g_{i, \varepsilon}^{l}\right) \text { for } 1 \leq i \leq k, \\
z_{j, \varepsilon}-\chi\left(z_{j, \varepsilon}\right) \text { for } 1 \leq j \leq t, \quad z_{j, \varepsilon}-\chi\left(z_{j, \varepsilon}\right) \text { for } t+1 \leq j \leq p .
\end{gathered}
$$

The number of irreducible representations with central character $\chi$ is equal to $l^{t}$. This proves $1)$.

To calculate subalgebras $\mathfrak{g}_{\chi}$ and $\mathfrak{g}_{\chi_{0}}$ we find generators of the subalgebras $G_{\chi}$ and $G_{\chi_{0}}$ of $\mathfrak{R}_{\varepsilon}$ :

$$
\begin{gathered}
G_{\chi}=<z_{j, \varepsilon}^{l}-\chi\left(z_{j, \varepsilon}^{l}\right), 1 \leq j \leq t ; z_{j, \varepsilon}-\chi\left(z_{j, \varepsilon}\right), t+1 \leq j \leq p ; \quad \mathfrak{i}> \\
G_{\chi 0}=<z_{j, \varepsilon}^{l}-\chi\left(z_{j, \varepsilon}^{l}\right), 1 \leq j \leq p ; \mathfrak{i}_{0}>
\end{gathered}
$$


We see $G_{\chi_{0}} \subset G_{\chi}$. This defines the homomorphism $\psi: \mathfrak{g}_{\chi_{0}} \rightarrow \mathfrak{g}_{\chi}$.

Since $\mathfrak{i}$ is an intersection of $\mathfrak{R}$-ideal $\mathfrak{I}$ with $\mathfrak{Z}_{\varepsilon}$, then $\mathfrak{i}$ is a Poisson ideal of $\mathfrak{Z}_{\varepsilon}$ [10, Lemma 3.12]. Similar for $\mathfrak{i}_{0}$. This implies 2). Denote

$$
e_{i}=z_{i \varepsilon}^{l}-\chi\left(z_{i \varepsilon}^{l}\right) \bmod m_{\chi}^{2}, \quad 1 \leq i \leq t \text { and } \mathfrak{t}=\operatorname{span}\left\{\mathrm{e}_{\mathrm{i}} ; 1 \leq \mathrm{i} \leq \mathrm{t}\right\} .
$$

The Lie algebra $\mathfrak{g}_{\chi}$ is a sum (as a linear space) of $\mathfrak{t}$ and $\mathfrak{n}$ spanned modulo $m_{\chi}^{2}$ by $z_{j, \varepsilon}-\chi\left(z_{j, \varepsilon}\right)$, $t+1 \leq j \leq p$ and $\mathfrak{n}^{\prime}$. Similarly, $\mathfrak{g}_{\chi_{0}}$ is a sum (as a linear space) of $\mathfrak{t}_{0}=\mathfrak{t}$ and $\mathfrak{n}_{0}$, spanned modulo $m_{\chi_{0}}^{2}$ by $z_{j, \varepsilon}^{l}-\chi\left(z_{j, \varepsilon}^{l}\right), t+1 \leq j \leq p$ and $\mathfrak{n}_{0}^{\prime}$.

Let us prove that $\mathfrak{n}$ is a nilpotent ideal in $\mathfrak{g}_{\chi}$. Similar for $\mathfrak{n}_{0}$. Any element of $\mathfrak{R} S_{*}^{-1}$ (is a sum of monomials of

$$
x_{1}^{n_{1}} \cdots x_{m}^{n_{m}} \tilde{x}_{m+1}^{n_{m+1}} \cdots \tilde{x}_{N}^{n_{N}}
$$

We define the degree $\operatorname{deg}(a):=\left(n_{m+1}, \ldots, n_{N}\right)$ of any monomial $a$. For any two monomials $a, b$ there exists $s \in \mathbb{Z}$ such that $a b-q^{s} b a$ is a sum of monomials of lower degree with respect to the lexicographical ordering. For any $A, B \in Z_{\varepsilon}$ we have $\{A, B\}=$ const $A B+$ $\{$ the lower terms $\}$. This verifies that $\mathfrak{n}$ is a nilpotent ideal.

Let us prove that the Lie subalgebra $\mathfrak{t}$ is diagonalizable. The elements $x_{1}, \ldots, x_{m}$ are FAelements in $\mathfrak{R}[9$, 11]. That is for any $1 \leq i \leq m$ and $a \in \mathfrak{R}$ there exists a polynomial $f(t)$ (with roots in $\left.\left\{q^{s}\right\}_{s \in \mathbb{Z}}\right)$ such that $f\left(\operatorname{Ad}_{x_{i}}(a)=0\right.$. The adjoint action $\operatorname{Ad}_{x_{i}}$ is diagonalizable [9]. One can choose $f(t)$ with different roots $q^{\gamma_{1}}, \ldots, q^{\gamma_{k}}$. The derivation $\mathcal{D}_{i}^{\prime}:=x_{\varepsilon}^{-l} \mathcal{D}_{x^{l}}: \mathfrak{R}_{\varepsilon} \rightarrow \mathfrak{R}_{\varepsilon}$ for $x=x_{i}$ obey $f_{1}\left(\mathcal{D}_{i}^{\prime}\right)\left(a_{\varepsilon}\right)=0$ where $f_{1}(t)$ is a polynomial with different roots $c \gamma_{1}, \ldots, c \gamma_{k}$, $c=l \varepsilon^{l-1}$. This imply that $\mathcal{D}_{i}^{\prime}$ is diagonalizable. The same is true for $z_{i}^{l}$. Finally, $\operatorname{ad}_{e_{i}}$ are simultaneously diagonalizable.

Definition 2.3. Let $R$ be domain with unit. Consider the set of pairs $\left\{\left(\mathcal{P}_{\mu}, S_{\mu}\right)\right\}$ where $S_{\mu}$ is a denominator subset $R$ and $\mathcal{P}_{\mu}$ is a prime ideal in $R$ (i.e $\mathcal{P}_{\mu} \in \operatorname{Spec}(R)$ ) with empty intersection with $S_{\mu}$. We call $\left\{\left(\mathcal{P}_{\mu}, S_{\mu}\right)\right\}$ a stratification of $\operatorname{Spec}(R)$ if for any $I \in \operatorname{Spec}(R)$ there exists a unique $\mu$ such that $I \supset \mathcal{P}_{\mu}$ and $I \cap S_{\mu}=\emptyset$. If $R$ is a free $C$-module over commutative ring $C$, we assume, in addition, that $I$ and any $\mathcal{P}_{\mu}$ have zero intersection with $C$.

Definition 2.4. Let $R_{q}$ be a quantum solvable algebras over $C:=\mathbb{C}\left[q, q^{-1}\right]$ and $\left\{\left(\mathcal{P}_{\mu}, S_{\mu}\right)\right\}$ be a stratification of $R_{q}$. We call $\left\{\left(\mathcal{P}_{\mu}, S_{\mu}\right)\right\}$ an admissible stratification if

1 ) for any $\mu$ there exists isomorphism $\theta_{\mu}: R_{q} S_{\mu}^{-1} \rightarrow \mathfrak{R}_{\mu}$ such that $\mathfrak{R}_{\mu}$ and $\mathfrak{I}_{\mu}:=\theta_{\mu}\left(\mathcal{P}_{\mu}\right)$ form a standard pair;

2) the stratification $\left\{\left(\mathcal{P}_{\mu}, S_{\mu}\right)\right\}$ admits specialization modulo $q-\varepsilon$ (i.e. $\left\{\left(\mathcal{P}_{\mu, \varepsilon}, S_{\mu, \varepsilon}\right)\right\}$ is a stratification of $\mathfrak{R}_{\varepsilon}$ ).

3) $S_{\mu, \varepsilon}:=S_{\mu} \bmod (q-\varepsilon) \subset Z_{0}$ and $\theta_{\mu}\left(S_{\mu}\right)$ is generated by $x_{1}^{l}, \ldots, x_{m}^{l}$.

Proposition 2.5. Let $R_{q}$ be a quantum solvable algebra and $l$ be admissible for $R_{q}$. Suppose that $x_{1, \varepsilon}^{l}, \ldots, x_{N, \varepsilon}^{l}$ lie in the center of $R_{\varepsilon}$ and generate a Poisson central subalgebra (denote $Z_{0}$ ). Suppose that $R_{q}$ has an admissible stratification $\left\{\left(\mathcal{P}_{\mu}, S_{\mu}\right)\right\}$. Then Main Theorem is true for $\chi$. Proof. Let $\chi \in \mathfrak{X}$. Choose $\mu$ such that $\chi\left(S_{\mu, \varepsilon}\right) \neq 0$ and $\chi$ is annihilated by $\mathfrak{i}_{\mu}$. Apply Proposition 2.2.

\section{Existence of admissible stratification}

To prove Main Theorem we present an admissible stratification for quantum algebras A1-A4. A1) The algebra of twisted polynomials. Let the matrices $\mathbb{Q}$ and $\mathbb{S}$ be as in the above. The algebra $R=A_{\mathbb{S}}$ of twisted polynomials is generated by $x_{1}, \ldots x_{n}, x_{n+1}^{ \pm 1}, \ldots, x_{N}^{ \pm 1}$ subject to the relations $x_{i} x_{j}=q_{i j} x_{j} x_{i}$. 
Choose some subset $T \subset \Lambda=\{1,2, \ldots, n\}$. Consider the ideal $\mathcal{P}_{T}$ generated by $\left\{x_{i}: i \in T\right\}$ and the denominator subset $S_{T}$ generated by $\left\{x_{i}^{l}: i \notin T\right\}$.

Proposition 3.1. Main Theorem is true for the Algebra of twisted polynomials.

Proof. The set of pairs $\left\{\left(\mathcal{P}_{T}, S_{T}\right)\right\}$ is an admissible stratification. By direct calculations, $\left\{a_{i}, a_{j}\right\}=c s_{i j} a_{i} a_{j}$ where $a_{i}=x_{i, \varepsilon}^{l}$ and $c=l \varepsilon^{l-1}$. Apply Proposition 2.5.

A2) Quantum Weyl algebra. Let $\mathbb{S}=\left(s_{i j}\right)_{i j}^{n}$ be skew-symmetric integer matrix and $q$ be indeterminate. As above we put $q_{i j}=q^{s_{i j}}$ and form the matrix $\left.\mathbb{Q}=\left(q_{i j}\right)_{i j=1}^{n}\right)$. Given non-zero integers $s_{1}, \ldots, s_{n}$ define

$$
q_{1}=q^{s_{1}}, \ldots, q_{n}=q^{s_{n}} .
$$

We consider two new matrices. The first matrix $\mathbb{P}=\left(\left(p_{i j}\right)_{i j=1}^{n}\right)$ with entries subject $p_{i i}=$ $p_{i j} p_{j i}=1$ and such that

$$
p_{i j}=q_{i} q_{i j}, \text { for } \mathrm{i}<\mathrm{j} .
$$

The second one $\left.\mathbb{R}=\left(r_{i j}\right)_{i j=1}^{n}\right)$ has entries

$$
r_{i j}=\left\{\begin{array}{l}
q_{j i}, \text { if } \mathrm{i}<\mathrm{j}, \\
q_{i}, \text { if } \mathrm{i}=\mathrm{j}, \\
p_{j i}=q_{j} q_{j i}, \text { if } \mathrm{i}>\mathrm{j} .
\end{array}\right.
$$

Form skew-symmetric integer matrix $\mathbb{T}=\left(t_{i j}\right)_{i j=1}^{n}$ such that $p_{i j}=q^{t_{i j}}$ and integer matrix $\mathbb{U}=\left(u_{i j}\right)_{i j=1}^{n}$ such that $r_{i j}=q^{u_{i j}}$. Form matrices

$$
\mathbb{Q}^{*}=\left(\begin{array}{cc}
\mathbb{Q} & -\mathbb{R} \\
\mathbb{R} & \mathbb{P}
\end{array}\right), \quad \mathbb{S}^{*}=\left(\begin{array}{cc}
\mathbb{S} & -\mathbb{U} \\
\mathbb{U} & \mathbb{T}
\end{array}\right)
$$

Definition 3.2. The Quantum Weyl algebra $W$ is generated by $y_{1}, \ldots, y_{n}, x_{1}, \ldots, x_{n}$ with the following relations $y_{i} y_{j}=q_{i j} y_{j} y_{i}, x_{i} x_{j}=p_{i j} x_{j} x_{i}, x_{i} y_{j}=r_{i j} y_{j} y_{i}$ for $i \neq j$ and

$$
x_{i} y_{i}=q_{i} y_{i} x_{i}+\sum_{k<i}\left(q_{k}-1\right) y_{k} x_{k}+1 .
$$

The algebra $W$ is an quantum solvable algebra over $\mathbb{C}\left[q, q^{-1}\right]$ with the system of exponents $s_{1}, \ldots, s_{n}$. Denote $h_{k}=y_{k} x_{k}$. The relations imply

$$
\begin{gathered}
\left(\begin{array}{c}
x_{i} \\
y_{i}
\end{array}\right) h_{k}=h_{k}\left(\begin{array}{c}
q_{i} x_{i} \\
q_{i}^{-1} y_{i}
\end{array}\right) \quad \text { for } i<k, \\
\left(\begin{array}{c}
x_{i} \\
y_{i}
\end{array}\right) h_{k}=h_{k}\left(\begin{array}{c}
x_{i} \\
y_{i}
\end{array}\right) \quad \text { for } i>k .
\end{gathered}
$$

For any $1 \leq i \leq n$ we denote $w_{i}=1+\sum_{k \leq i}\left(q_{k}-1\right) y_{k} x_{k}$.

One can rewrite $(3.1)$ as follows $x_{i} y_{i}=q_{i} y_{i} x_{i}+w_{i-1}$. The variables $x_{i}, y_{i}, w_{i}$ obey the relations

$$
y_{i} w_{j}=\left\{\begin{array}{l}
q_{i}^{-1} w_{j} y_{i}, \text { for } i \leq j, \\
w_{j} y_{i}, \text { for } i>j,
\end{array}, \quad x_{i} w_{j}=\left\{\begin{array}{l}
q_{i} w_{j} x_{i}, \text { for } i \leq j, \\
w_{j} x_{i}, \text { for } i>j,
\end{array} .\right.\right.
$$

By definition, $\varepsilon$ is an admissible $l$ th root of unity if $l$ is relatively prime with all principal minors of $S^{*}$ and with $s_{1}, \ldots, s_{n}$. The elements $a_{i}:=x_{i, \varepsilon}^{l}, b_{i}:=y_{i, \varepsilon}^{l}$ lie in the center of $Z_{\varepsilon}$ of $W_{\varepsilon}$ and generate the central subalgebra $Z_{0}$.

Denote $f_{i}=w_{i, \varepsilon}^{l}, 1 \leq i \leq n$. Similarly to [13], one can prove that there exists the chain of non-zero complex numbers $\gamma_{1}, \ldots, \gamma_{n-1}$ such that

$$
f_{i}=1+\sum_{k<i} \gamma_{k} a_{k} b_{k}
$$


This implies that $f_{i} \in Z_{0}$. By direct calculations, $\left\{a_{i}, a_{j}\right\}=\gamma t_{i j} a_{i} a_{j},\left\{b_{i}, b_{j}\right\}=\gamma s_{i j} b_{i} b_{j}$, $\left\{a_{i}, b_{j}\right\}=\gamma u_{i j} a_{i} b_{j},\left\{a_{i}, b_{i}\right\}=\gamma s_{i} a_{i} b_{i}+f_{i-1}$ where $\gamma=l^{2} \varepsilon^{-1}$. We see that $Z_{0}$ is a Poisson subalgebra in $Z_{\varepsilon}$.

Denote $\Lambda=\{1, \ldots, n\}$. We shall call a triple $T=\left(T_{1}, T_{2}, T_{3}\right)$ of subsets of $\Lambda$ an admissible triple if $T_{1} \subseteq T_{2} \subseteq T_{3}$ and the following property holds: if $i \in T_{2}$ then $i$ and $i-1 \in T_{3}$.

Consider the ideal $\mathcal{P}_{T}$ of $W$ generated by $x_{i}, y_{j}$ and $w_{k}$ with $i \in T_{1}, j \in T_{2}$ and $k \in T_{3}$. Form the denominator subset $S_{T}$ generated by the following $q$-commuting elements $\left\{x_{i}^{l}, i \in T_{2}-T_{1}\right\}$, $\left\{y_{i}^{l}, i \in \Lambda-T_{2}\right\}$ and $\left\{w_{i}^{l}, i \in \Lambda-T_{3}\right\}$. The subset $S_{T}$ has empty intersection with $\mathcal{P}_{T}$. The set of pairs $\left\{\left(\mathcal{P}_{T}, S_{T}\right)\right\}$ is a stratification of $W$ (see [15, 16]).

Proposition 3.3. Main Theorem is true for the Quantum Weyl algebra.

Proof. The set of pairs $\left(\mathcal{P}_{T}, S_{T}\right)$ is an admissible stratification.

A3-A4. Cases of algebras $U_{q}(\mathfrak{b})=\mathbb{C}_{q}[B]$ and $\mathbb{C}_{q}[\mathrm{G}]$. Let $\mathfrak{g}$ be a semisimple Lie algebra with the system of simple roots $\alpha_{1}, \ldots, \alpha_{n}$. Let $\mathrm{G}$ be its simply connected Lie group. Denote $d_{i}:=\frac{\left(\alpha_{i}, \alpha_{i}\right)}{2}$ and $C:=\mathbb{C}\left[q, q^{-1},\left(q^{d_{i}}-q^{-d_{i}}\right)^{-1}\right]$. The quantum universal enveloping algebra is an Hopf algebra over $C$ generated by $E_{i}, F_{i}, K_{i}^{ \pm 1}, 1 \leq i \leq n$ obeying Drinfeld-Jimbo relations. The algebra $\mathbb{C}_{q}[\mathrm{G}]$, the subalgebra of the dual Hopf algebra for $U_{q}(\mathfrak{g})$, is generated by matrix entries of irreducible finite dimensional representations $c_{f, v}(a):=f(a v), v \in V, f \in V^{*}, a \in U_{q}(\mathfrak{g})$.

We assume that $l$ is admissible. In the case of algebras A3-A4: $l$ is admissible if $l$ is odd and $l \geq 3$ in the case $G$ has $\mathrm{G}_{2}$ components. The algebra $C_{\varepsilon}[\mathrm{G}]$ has a central Poisson subalgebra $Z_{0}$ that is isomorphic to $\mathbb{C}[G]$ with the standard Belavin-Drinfeld bracket [3]. The algebra $\mathbb{C}_{q}[\mathrm{G}]$ has a subalgebra $R_{q}^{+}$generated by matrix entries $c_{f, v_{-\lambda}}$ where $v_{-\lambda}$ is the vector of lowest weight in the irreducible representation $V_{\lambda}$ with highest weight $\lambda$. The algebra $R_{q}^{+}$is isomorphic to $\mathbb{C}_{q}[\mathrm{~B}]$ where $\mathrm{B}:=\mathrm{B}^{+}$. By Drinfeld pairing the algebra $\mathbb{C}_{q}[\mathrm{~B}]$ is isomorphic to $U_{q}\left(\mathfrak{b}^{-}\right)$.

Proposition 3.4. Main theorem is true for the algebras A3-A4.

Proof. First, notice that the algebra $\mathbb{C}_{q}[\mathrm{G}]$ has the denominator subset $S$ generated by matrix entry $c_{\rho, v_{-} \rho}$ where $\rho$ equal to the half of sum of positive roots. The localization $\mathbb{C}_{q}[\mathrm{G}] S^{-1}$ is isomorphic to the subalgebra in $U_{q}\left(\mathfrak{b}^{-}\right) \otimes U_{q}\left(\mathfrak{b}^{+}\right)$generated by $K_{\lambda} \otimes K_{-\lambda}, F_{i} \otimes 1,1 \otimes E_{i}$, $1 \leq i \leq n$. It suffices to construct an admissible stratification for $\mathbb{C}_{q}[\mathrm{~B}]$.

The algebra $R_{q}^{+}$(that is equal to $\mathbb{C}_{q}[\mathrm{~B}]=U_{q}\left(\mathfrak{b}^{-}\right)$) has an stratification $\left(\mathcal{P}_{w}, S_{w}\right)$ where $w$ is an element of the Weyl group $W$ [3, 5, 18]. By definition, the ideal $\mathcal{P}_{w}$ is generated (as ideal) by the elements $c_{f, v_{-\lambda}}$ where $f$ is orthogonal to subspace $U_{q}\left(\mathfrak{b}^{-}\right) t_{w} v_{-\lambda}$ (here $t_{w}$ is the corresponding element of the braid group). The denominator subset $S_{w}$ is generated by the element $z_{w}:=c_{w f^{\rho}, v_{-}}$where $f^{\rho}$ is the element of highest weight in $V_{\rho}^{*}$.

Below we present the other construction of pair $\left(\mathcal{P}_{w}, S_{w}\right)$. Decompose the element $w_{0}$ (of highest length in the $W$ ) into product of simple reflections

$$
w_{0}=s_{1} \ldots s_{k} s_{k+1} \ldots s_{N}, \quad s_{t}:=s_{\alpha_{i_{t}}}
$$

such that $w=s_{1} \ldots s_{k}$. Denote $w_{t}=s_{1} \ldots s_{t}$ (here $\left.w_{k}=w\right)$ and $z_{t}=z_{w_{t}}$. The elements $z_{i}$ are $q$-commute [5, Cor. 3.2]. As usual denote $\beta_{t}:=s_{1} \ldots s_{t-1}\left(\alpha_{i_{t}}\right)$. The algebra $R_{q}^{+}$is a quantum solvable algebra with respect to the chain of generators

$$
K_{1}^{ \pm 1}, \ldots, K_{n}^{ \pm 1}, F_{\beta_{1}}, \ldots, F_{\beta_{1}}, \ldots, F_{\beta_{N}} .
$$

We denote by $B_{t}$ the subalgebra generated by $K_{1}^{ \pm 1}, \ldots, K_{n}^{ \pm 1}, F_{\beta_{k}}, \ldots, F_{\beta_{t}}$. We obtain the filtration $B_{1} \subset \ldots \subset B_{k} \subset B_{N}=R_{q}^{+}$. The subalgebra $B_{k}$ depends only on $w$ (denote $B_{w}:=B_{k}$ ) [5]. The element $z_{t}$ lies in $B_{t}$ and don't lie in $B_{t-1}$ [5, Lemma 3.2]. Denote by $S_{t}$ the denominator subset generated by $z_{t}$. The ideal $\mathcal{P}_{w}$ has zero intersection with $B_{k}$ and $B_{k} S_{k}^{-1}=R_{q}^{+} S_{k}^{-1} / \mathcal{P}_{w} S_{k}^{-1}$. Let $S_{w}$ be the denominator subset generated by $S_{t}, 1 \leq t \leq k$ and 
$\mathfrak{R}_{w}:=R_{q}^{+} S_{w}^{-1}$. The elements $z_{t}$ are FA-elements in quantum solvable algebra $\mathfrak{R}_{w}$ [9, 11]. The adjoint action $\operatorname{Ad}_{z_{i}}$ are diagonalizable. Choose the new generators $z_{1}^{ \pm 1}, \ldots, z_{k}^{ \pm 1}, \tilde{F}_{\beta_{k+1}}, \ldots, \tilde{F}_{\beta_{N}}$ in localization $R_{q}^{+} S_{*}^{-1}$ (for definition of $S_{*}$ see section 2). The ideal $\mathcal{P}_{w} S_{*}^{-1}$ is generated by $\tilde{F}_{\beta_{t}}$ $k+1 \leq t \leq N$. The elements $z_{t, \varepsilon}^{l}$ lie in $Z_{0}$ [5, Theorem 1.6]. The pair $\left(R_{q}^{+}, \mathcal{P}_{w}\right)$ is a standard pair and the stratification $\left(\mathcal{P}_{w}, S_{w}\right)$ is an admissible stratification. This verifies the statement for $\mathbb{C}_{q}[\mathrm{~B}]$ (and therefore for $\mathbb{C}_{q}[\mathrm{G}]$ ).

\section{References}

[1] De Concini C., Kac V. G. Representations of quantum groups at roots of 1, Colloque Dixmier 1989, Progress in Math., 1990, V.92, 471-506,

[2] De Concini C., Kac V. G., Procesi C. Quantum coadjoint action, Journal of Amer.Math.Soc., 1992, V. 5, 151-189.

[3] De Concini C., Lyubashenko V. Quantum function algebra at roots of 1, Advances in Math., V.108, 1994, 205-262.

[4] De Concini C., Procesi C. Quantum Groups, Lecture Notes in Math., 1993, V.1565, 31140 .

[5] De Concini C., Procesi C. Quantum Schubert cells and representations at roots of 1, Algebraic groups and Lie groups(G.I.Lehrer,editor), N9 in Australian Math.Soc.Lecture Series, Cambridge University press, Cambridge,1997.

[6] Goodearl K. R., Prime Spectra of Quantized Coordinate Rings, math.QA/9903091, Lecture Notes in Pure and Appl.Math, 2000, '.210, 205-237.

[7] Brown K. A., Gordon I. Poisson orders, symplection reflection algebras and representation theory, preprint math.RT/0201042, J. reine angew. Math., V.559, 2003, 193-216.

[8] M.V.Karasev, V.P.Maslov Nonlinear Poisson brackets. Geometry and quantization, Moscow, Nauka, 1991(russian)

[9] Panov A. N. Fields of fractions of Quantum solvable algebras, J.Algebra, 2001, V.236, P.110-121.

[10] Panov A. N. Quantum solvable algebras. Ideals and representations at roots of 1, Transformation groups, 2002, V.7, N4, 379-402.

[11] Panov A. N. Irreducible representations of quantum solvable algebras at roots of 1, Algebra and analysis, V.15, 2003, N4, P.229-259(russian).

[12] McConnel J. C., Robson J. C. Noncommutative Noetherian Rings, Wileys-Interscience, New York, 1987.

[13] Jakobsen H., Zhang H., Quantized Heisenberg spaces, Algebras and Representation Theory, V.2, N2, 2000, P.151-174

[14] Zhang H. The Irreducible Representations of the Coordinate Ring of the Quantum Matrix Space, Algebra Colloquium, V.9:4, 2002, P.383-392 
[15] Oh Sei-Qwon Primitive ideals of the coordinate ring of quantum symplectic space, J.Algebra, 1995, V.174, 531-552

[16] Horton K.L. The prime and primitive spectra of multilinear quantum symplectic and euclidean spaces, Comm.Algebra, V.31, 2003, N.10, 4713-4743

[17] Cauchon G. Effacement des derivations et spectres primiers des algebras quantiques, J.Algebra, 2003, V.260, N2, 476-518.

[18] Joseph A. Quantum groups and their primitive ideals, Berlin, Heidelberg, Springer-Verlag, 1995 